\title{
Sensory analysis of calcium-biofortified lettuce
}

Sunghun Park ${ }^{1, *}$, Mark P. Elless², Jungeun Park' ${ }^{1}$ Alicia Jenkins ${ }^{3}$, Wansang Lim', Edgar Chambers IV and Kendal D. Hirschi ${ }^{4,5}$

'Department of Horticulture, Forestry and Recreation Resources, Kansas State University, Manhattan, KS 66506, USA

2Edenspace Systems Corporation, 3810 Concorde Parkway, Chantilly, VA 20151, USA

${ }^{3}$ Sensory Analysis Center, Department of Human Nutrition, Kansas State University, Manhattan, KS 66506, USA

${ }^{4}$ Vegetable and Fruit Improvement Center, Texas A \& M University, College Station, TX 77845, USA

${ }^{5}$ Baylor College of Medicine, USDAIARS Children's Nutrition Research Center, 1100 Bates St., Houston, TX 77030, USA

Received 16 May 2008; revised 2 September 2008; accepted 9 September 2008.

*Correspondence (fax 001 (785) 532-6949; e-mail shpark@ksu.edu)

Keywords: biofortification, calcium, $\mathrm{H}^{+}$/ $\mathrm{Ca}^{2+}$ transporter, lettuce, nutrition, sensory analysis.

\begin{abstract}
Summary
Vegetables represent an attractive means of providing increased calcium nutrition to the public. In this study, it was demonstrated that lettuce expressing the deregulated Arabidopsis $\mathrm{H}^{+} / \mathrm{Ca}^{2+}$ transporter sCAX1 (cation exchanger 1) contained 25\%-32\% more calcium than controls. These biofortified lettuce lines were fertile and demonstrated robust growth in glasshouse growth conditions. Using a panel of highly trained descriptive panellists, biofortified lettuce plants were evaluated and no significant differences were detected in flavour, bitterness or crispness when compared with controls. Sensory analysis studies are critical if claims are to be made regarding the efficacy of biofortified foods, and may be an important component in the public acceptance of genetically modified foods.
\end{abstract}

\section{Introduction}

The Dietary Reference Intakes for calcium are set at levels with desirable retention of body calcium because a high bone density decreases the incidence of bone fractures. Osteoporosis, a condition of reduced bone density, is an underlying cause of bone fragility, especially among women. In the USA, $15 \%$ of calcium intake is provided by green vegetables and dried fruits (National Academy of Sciences, 1999; Guegen and Pointillart, 2000). Few vegetables are 'good' sources of calcium, and these seemingly 'good' vegetables do not constitute a significant part of the average diet in the USA. In contrast, a popular vegetable, such as lettuce, provides only a small fraction of the Dietary Reference Intake for calcium (United States Department of Agriculture, 2004). Plant scientists have adopted a strategy, now known as biofortification (Bouis, 2003; Pfeiffer and McClafferty, 2007), which attempts to develop genetically improved varieties that can achieve enhanced nutrient densities in their edible parts. In this study, we describe a means to biofortify lettuce with increased calcium content. Lettuce is an attractive dietary option for enhancing the consumption of dietary calcium. Lettuce is rich in vitamin $\mathrm{K}$ (as much as $167 \mu \mathrm{g} / \mathrm{head}$; United States Department of Agriculture, 2004), and its daily consumption has been found to significantly reduce the risk of hip fractures in women in comparison with women who consume lettuce at a lower rate (Feskanich et al., 1999). Therefore, lettuce is already combating osteoporosis and the addition of calcium is expected to produce synergistic benefits. A large portion of women in the US eat lettuce daily (United States Department of Agriculture, 2000), suggesting that biofortified lettuce will be consumed by the target audience for the prevention of osteoporosis.

Plant transporters can be engineered for increased bioavailable calcium content in agriculturally important crops (Park et al., 2004; Shigaki and Hirschi, 2006; Morris et al., 2008). The $\mathrm{Ca}^{2+} / \mathrm{H}^{+}$antiporters, termed $\mathrm{CAX}$ (for cation exchangers), located on the vacuolar membrane are important for calcium sequestration. This led to the idea that an engineered version of a plant $\mathrm{Ca}^{2+} / \mathrm{H}^{+}$antiporter, sCAX1, could be used for biofortification, by increasing the calcium levels of edible roots, such as carrots. Modified carrots expressing high levels of sCAX1 accumulate almost twofold more calcium in the edible part compared with control plants, 
without perturbing growth, development or fertility, under controlled laboratory conditions. Feeding trials using these labelled carrots demonstrated that the total amount of calcium absorbed was significantly increased in both mice and humans with diets containing the modified carrots (Morris et al., 2008). However, the taste and tactile components of the carrots were not measured.

Although biofortification efforts are attempting to enhance the nutritional quality of numerous foods (Park et al., 2005a; Diaz de la Garza et al., 2007), few studies have been performed to assess how genetically engineered foods alter the taste qualities and consumer perceptions of the foods. Calcium in the form of a water-soluble salt or complex can be added to food and/or beverages, but may cause a bitter taste (Lawless et al., 2003). Thus, it is important to evaluate how sCAX1-expressing plants taste. Sensory analysis applies the principles of experimental design and statistical analysis to the use of human senses for the purposes of evaluating a product. This discipline requires panels of human assessors, on whom the products are tested, and the recording of the responses made by them. By applying sensory analysis to genetically modified foods, we can begin to determine whether these products are commercially viable.

Given the scope of dietary calcium deficit in consumers, it is probable that enhanced calcium content in popular vegetables, such as lettuce, could have a positive impact on calcium consumption. In this study, we demonstrate increased calcium levels in the edible portion of sCAX1-expressing lettuce with no negative impact on lettuce yields. Using a panel of five highly trained descriptive panellists, we also evaluated the flavour, bitterness and crispness of the biofortified lettuce. Sensory evaluation studies represent an important component in the experimental gauntlet that must be navigated by biofortified foods.

\section{Results}

\section{sCAX1 expression in lettuce}

Ectopic expression of the deregulated Arabidopsis sCAX1 transporter causes increased calcium levels in tobacco, carrots, potatoes and tomatoes (reviewed in Shigaki and Hirschi, 2006). The sCAX1-expressing tobacco and tomato lines exhibit certain deleterious phenotypes (Hirschi, 1999; Park et al., 2005b). In this study and previous reports, in order to alleviate the potential ill effects caused by sCAX1 expression using the cauliflower mosaic virus (CaMV) 355 promoter (Hirschi, 1999), we opted to also express sCAX1 under the control of the cell division cycle (cdc2a) promoter (Doerner et al., 1996).
In Arabidopsis, cdc2a transcript levels are correlated with the competence to divide (Doerner et al., 1996); however, the expression of this Arabidopsis promoter has not been detailed in lettuce. In previous studies, we have found that sCAX1expressing tobacco plants driven by the cdc2a promoter (CDCCAX) express less than one-half the amount of sCAX1 RNA when compared with sCAX1-expressing plants driven by the constitutively expressed CaMV 35 S promoter (35SCAX) (Park et al., 2004). In lettuces, we generated 20 35SCAXexpressing lines and 24 CDCCAX-expressing lines.

We randomly selected and confirmed 11 morphologically normal transgenic lettuce lines by Southern blot analysis. Genomic DNA was digested with ECoRI (for CDCCAX transgenic lines) and Xbal (for 35SCAX transgenic lines) (yielding border fragments which include a portion of the inserted T-DNA and genomic DNA) and hybridized with the sCAX1 probe. Digestion of the lettuce genomic DNA with these restriction enzymes revealed the transgene copy number from the number of hybridizing bands and independent transformation events from the hybridization patterns. As demonstrated in Figure 1a (parts of the data are shown), 35SCAX and CDCCAX lines contain various copy numbers of the sCAX1 expression vector. The strong band at the top (lane CDCCAX-13) may have resulted from an excess of loaded genomic DNA. RNA gel blots document that SCAX1 transcripts accumulated in all of the transgenic lines but not in the controls (Figure 1b, parts of the data are shown). The inability to detect an SCAX1 homologue in the wild-type (control) lines by Southern or Northern analysis may be the result of the stringency of hybridization and membrane washing used in this study. Our inability to identify the endogenous lettuce genomic CAX1 could be a result of the limited hybridization targets, given that a cDNA probe was used. Indeed, the $4.1 \mathrm{~kb}$ of Arabidopsis genomic CAX1 DNA contains nine introns (Hirschi, 1999). Regardless, using Southern analysis, three independent primary transgenic lines demonstrated either a single-copy insertion (35SCAX-4) or low-copy insertion events (35SCAX-3 and CDCCAX-15) (Figure 1a), with each line displaying high sCAX1 expression levels (Figure 1b, parts of the data are shown). These three sCAX1-expressing lines were selected for further characterization in subsequent generations.

\section{Phenotypes of sCAX1-expressing lettuce}

In both the 35SCAX- and CDCCAX-expressing plants, deregulated expression of sCAX1 did not alter the morphology or growth characteristics of the lettuce (Figure 2a). Furthermore, we were unable to measure any calcium oxalate crystals, an 
(a)

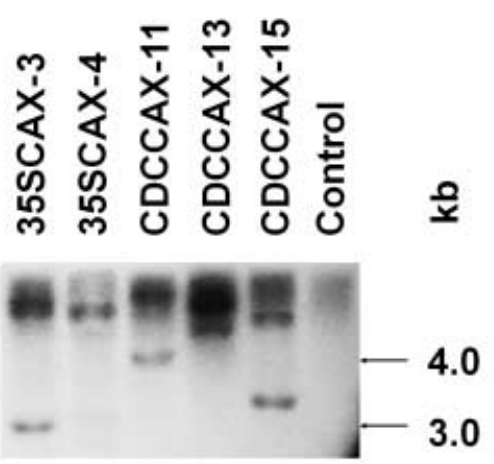

(b)
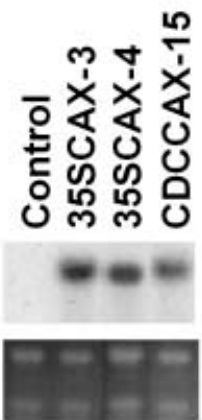

sCAX1

rRNA

Figure 1 Molecular analyses of primary transgenic lettuce plants. (a) Southern blot analysis of transgenic lettuces. Five to ten micrograms of lettuce genomic DNA were digested with Xbal (for 35SCAX) or ECoRI (for CDCCAX), and hybridized with the SCAX1 CDNA probe. Lanes 35SCAX-3 and 35SCAX4, transgenic lettuces with the pCaMV35S::sCAX1 vector construct; lanes CDCCAX-11, CDCCAX-13 and CDCCAX-15, transgenic lettuces with the pcdc2A::sCAX1 vector construct; lane Control, wild-type lettuce. Arrows indicate the expected fragments larger than $2.5 \mathrm{~kb}$ corresponding to the integration of T-DNA into the lettuce genomic DNA. (b) Northern blot analysis of transgenic lettuces. Seven micrograms of total RNA from expanded leaves were hybridized with the SCAX1 cDNA probe. Ethidium bromide-stained rRNA (bottom) is shown as a loading control.
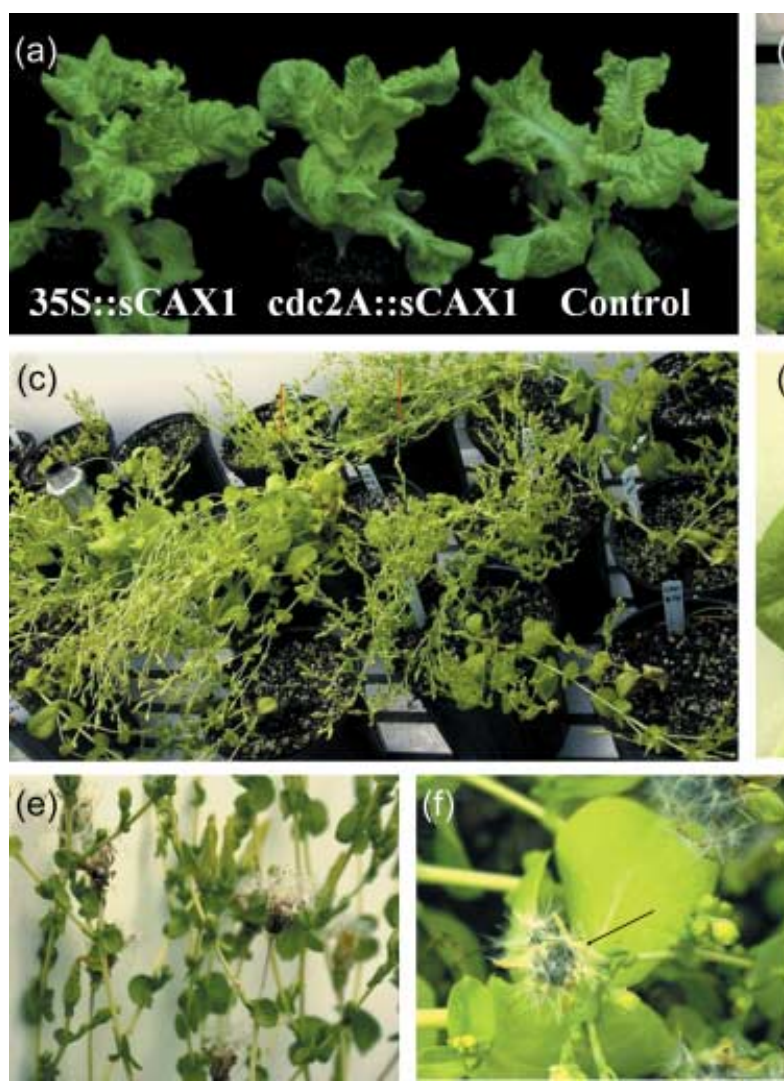

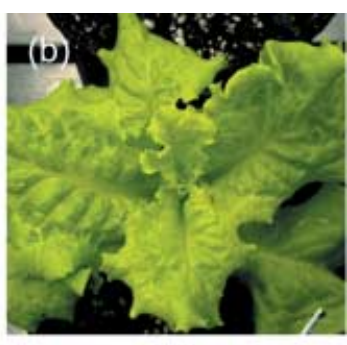

(d)
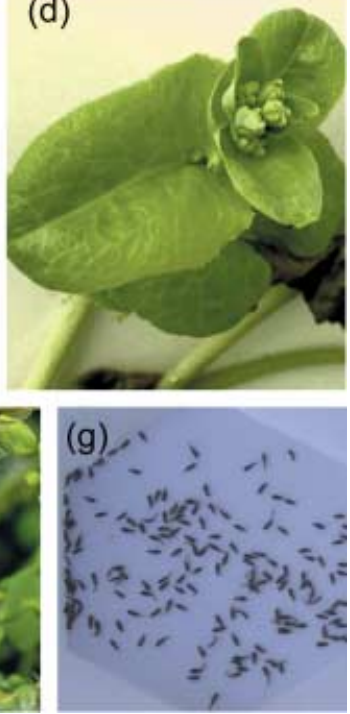

Figure 2 Phenotype of primary transgenic SCAX1-expressing lettuce plants. (a-c) Morphology and growth characteristics of sCAX1-expressing lettuce plants. (d-g) Seed set and seeds of sCAX1-expressing lettuce plants. sCAX1 expression did not perturb the morphology, growth or seed set (a-f), and all sCAX1-expressing lettuce lines were capable of making viable seeds (g). Red arrows indicate control plants. Dark arrow indicates a seed set. anti-nutrient of calcium, in either the control or SCAX1-expressing plants (data not shown). Although the sCAX1-expressing tobacco and tomato lines display calcium deficiencylike symptoms that are suppressed by the addition of calcium (Hirschi, 1999; Park et al., 2005b), the sCAX1-expressing lettuce lines were not sensitive to calcium deficiency and did not require any additional calcium supplementation for normal growth. In addition, both sCAX1-expressing lines and controls grew similarly on Murashige and Skoog (MS) medium (Murashige and Skoog, 1962) (data not shown). Regardless of the expression construct used, sCAX1 expression did not perturb the morphology, growth (Figure 2b) or seed set (Figure 2c,d). Moreover, the seed set was not delayed, and all sCAX1-expressing lettuce lines were capable of making viable seeds (Figure 2e). 


\section{Calcium accumulation in $\mathrm{s} C A X 1$-expressing $\mathrm{T}_{1}$ generation lettuce}

Initially, to determine whether $T_{1}$ lettuce contained sCAX1 genes, polymerase chain reaction (PCR) analysis was conducted to amplify the sCAX1 gene sequence (640-bp fragment). Figure 3a shows the analysis of PCR amplification of genomic DNA from three selected kanamycin-resistant lettuce lines. The morphology and growth characteristics of sCAX1expressing $T_{1}$ lettuce were indistinguishable from those of controls (Figure 3b). Total accumulation of calcium and other ions was measured in the edible portions of the $T_{1}$ lettuce lines. There was variability in the calcium content of the $T_{1}$ transgenic lettuce; however, most of the sCAX1-expressing lines accumulated more calcium (27\%-29\%) than controls $(14.7 \pm 0.4 \mathrm{mg} / \mathrm{g}$ dry weight for control lines, $18.6 \pm 1.0 \mathrm{mg} /$ $\mathrm{g}$ dry weight for 35SCAX lines and $18.9 \pm 1.4 \mathrm{mg} / \mathrm{g}$ dry weight for $C D C C A X$ lines; Figure $3 c)$. We were interested to determine whether SCAX1-expressing lettuce demonstrated an increased content of other minerals; however, no significant increase in other minerals $\left(\mathrm{Cu}^{2+}, \mathrm{Fe}^{2+}, \mathrm{Mn}^{2+}\right.$ and $\left.\mathrm{Zn}^{2+}\right)$ was observed with any of the lines analysed (data not shown). We attribute these phenotypes to sCAX1 expression in agreement with our previous observations in other transgenic plants, where vector control lines and lines expressing nonfunctional CAX transporters consistently contained mineral levels similar to those of wild-type controls (Shigaki and Hirschi, 2006).

\section{Phenotypes, yield and calcium accumulation in sCAX1- expressing $\mathrm{T}_{2}$ generation lettuce}

To confirm that the increased calcium accumulation was consistent in $T_{2}$ generation lettuce, three $T_{1}$ transgenic lines showing a single-copy insertion (35SCAX-4) and multiplecopy insertions (35SCAX-3 and CDCCAX-15) from Southern analysis (Figure 1a, parts of the data are shown) were selected, and subjected to further evaluation of the phenotypes, yield and calcium accumulation in sCAX1-expressing $T_{2}$ generation lettuce lines. Initially, reverse transcriptase (RT)PCR analysis with Arabidopsis SCAX1-specific primers confirmed that SCAX1 was transcribed in all of the $T_{2}$ generation transgenic lines (Figure 4a). The morphology and growth characteristics of sCAX1-expressing $T_{2}$ lettuce were indistinguishable from those of controls (Figure 4b). Furthermore, the total yield (as measured by the fresh weight of the lettuce leaves at 60 days after germination) of the $T_{2} S C A X 1$-expressing lines was not significantly different from that of controls (Figure 4c). The total accumulation of calcium and other ions (a)

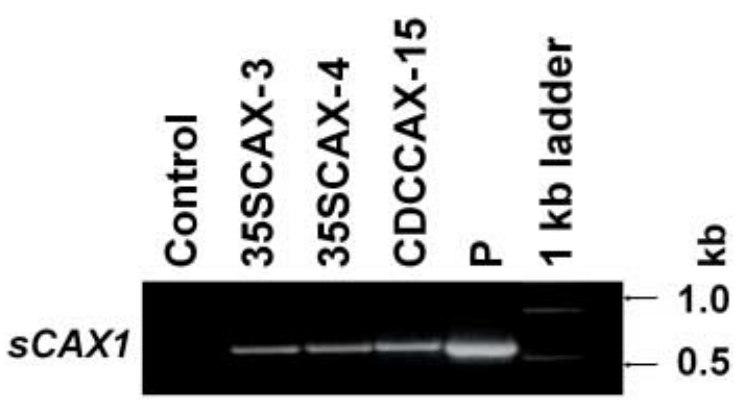

(b)

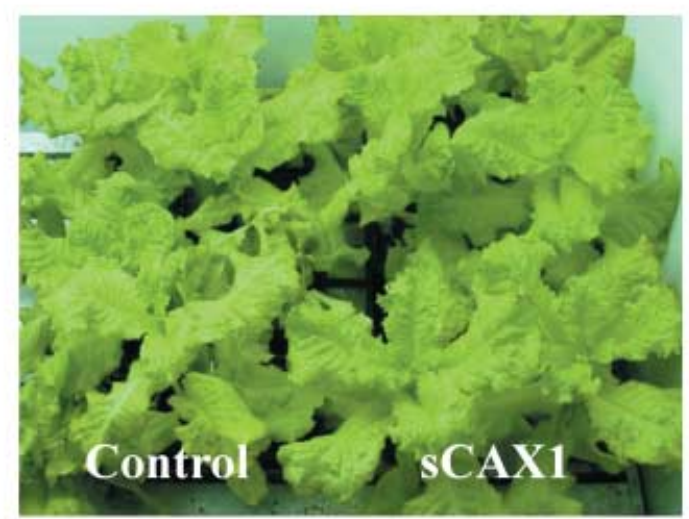

(c)

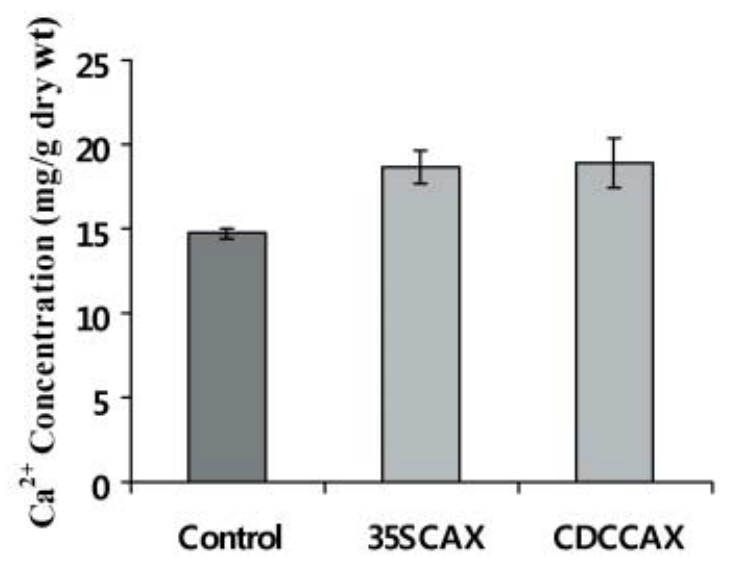

Figure 3 Phenotype, polymerase chain reaction (PCR) analysis and calcium accumulation in $\mathrm{SCAX}_{1}$-expressing $\mathrm{T}_{1}$ transgenic lettuce plants. (a) PCR analysis of $T_{1}$ transgenic lettuce plants. Expected 640-bp DNA fragment corresponding to the $S C A X 1$ gene sequence was amplified from kanamycin-resistant $T_{1}$ transgenic lettuce plants. Lane Control, negative control (wild-type lettuce); lanes 35SCAX-3 and 35SCAX-4, transgenic $T_{1}$

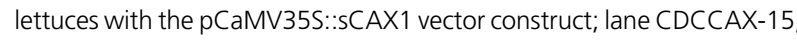
transgenic $\mathrm{T}_{1}$ lettuce with the $\mathrm{pcdc} 2 \mathrm{~A}:: \mathrm{s} \mathrm{CAX} 1$ vector construct; Lane $\mathrm{P}$, positive control (plasmid DNA). (b) The morphology and growth characteristics of sCAX1-expressing $\mathrm{T}_{1}$ transgenic lettuce plants are indistinguishable from those of wild-type controls. (c) Calcium accumulation in SCAX1-expressing $\mathrm{T}_{1}$ transgenic lettuce plants. The total calcium content of lettuce leaves was determined using an inductively coupled plasma emission spectrophotometer. Data are presented as the means \pm standard deviation of eight 35SCAX, eight CDCCAX and four control lines. 
(a)

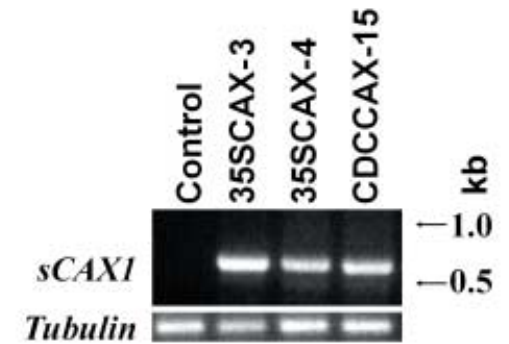

(c)

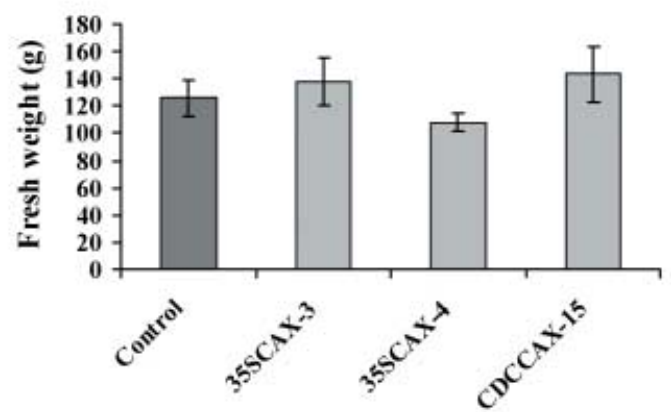

(d)

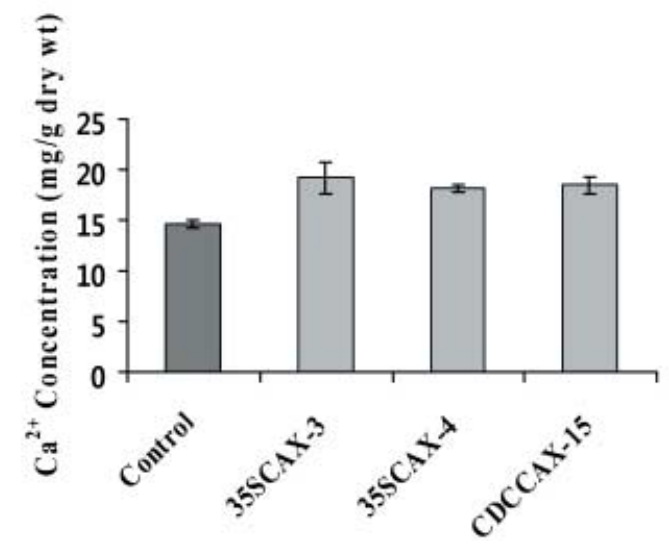

was measured in the edible portions of the $T_{2}$ lettuce lines. All of the sCAX1-expressing lettuce lines contained significantly more calcium (25\%-32\%) than controls $(14.5 \pm 0.4 \mathrm{mg} / \mathrm{g}$ dry weight for control line, $19.2 \pm 1.7 \mathrm{mg} / \mathrm{g}$ dry weight for 35SCAX-3 line, $18.1 \pm 0.3 \mathrm{mg} / \mathrm{g}$ dry weight for 35SCAX-4 line and $18.4 \pm 0.8 \mathrm{mg} / \mathrm{g}$ dry weight for CDCCAX-15 line; Figure $4 d)$. No significant increase in other minerals $\left(\mathrm{Cu}^{2+}\right.$, $\mathrm{Fe}^{2+}, \mathrm{Mn}^{2+}$ and $\mathrm{Zn}^{2+}$ ) was observed with any of the $\mathrm{T}_{2}$ lines analysed (data not shown).

\section{Sensory evaluation}

To determine whether there were any significant taste or flavour differences between SCAX1-expressing biofortified lettuce and controls, three $T_{2}$ transgenic lines (35SCAX-3,
35SCAX-4 and CDCCAX-15) containing significantly more calcium than controls (Figure 4d) were selected, and sensory analysis was carried out by a panel of five highly trained descriptive panellists. No significant differences were observed between the two groups (control or biofortified), indicating that no differences in flavour, bitterness or crispness were detected when the sCAX1-expressing biofortified lettuce was compared with controls (Table 1).

Table 2 shows a comparison of the sCAX1-expressing biofortified lettuce lines individually. Minor differences were found in two attributes. Line 15 (CDCCAX-15) of the biofortified lettuce group was found to be better than other lines (control and line 3) for a green, grassy/leafy flavour $(P<0.05)$. and line 3 (35SCAX-3) was better than the other transgenic lines for umami flavour $(P<0.05)$. Both attributes were similar to 
Table 1 Attributes and mean scores* of sCAX1-expressing calcium-biofortified lettuce lines and the control line

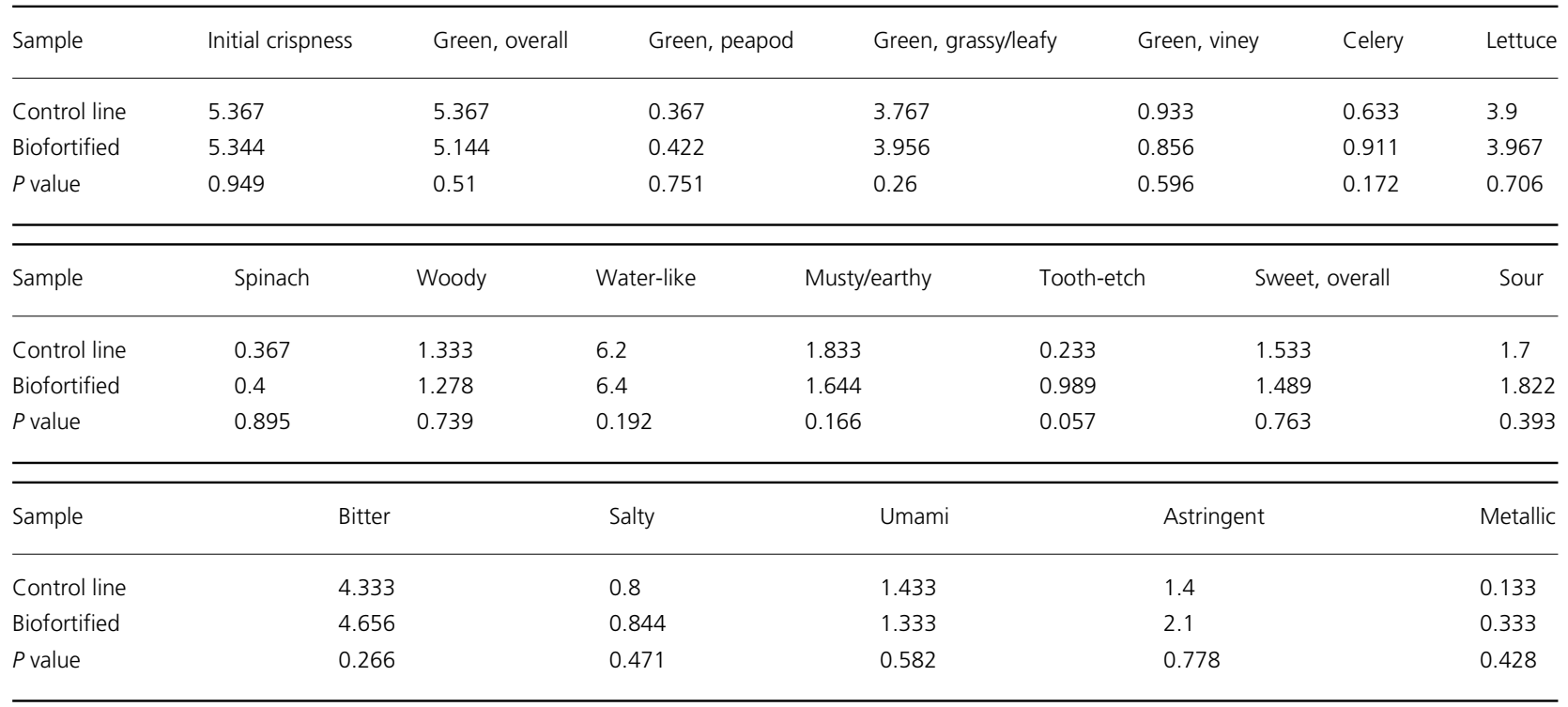

*Mean intensities based on a 15-point scale: 0 , none; 15, extremely strong.

Table 2 Attributes and mean scores* of sCAX1-expressing calcium-biofortified lettuce lines (line 3, 35SCAX-3; line 4, 35SCAX-4; line 15, CDCCAX-15) and the control line

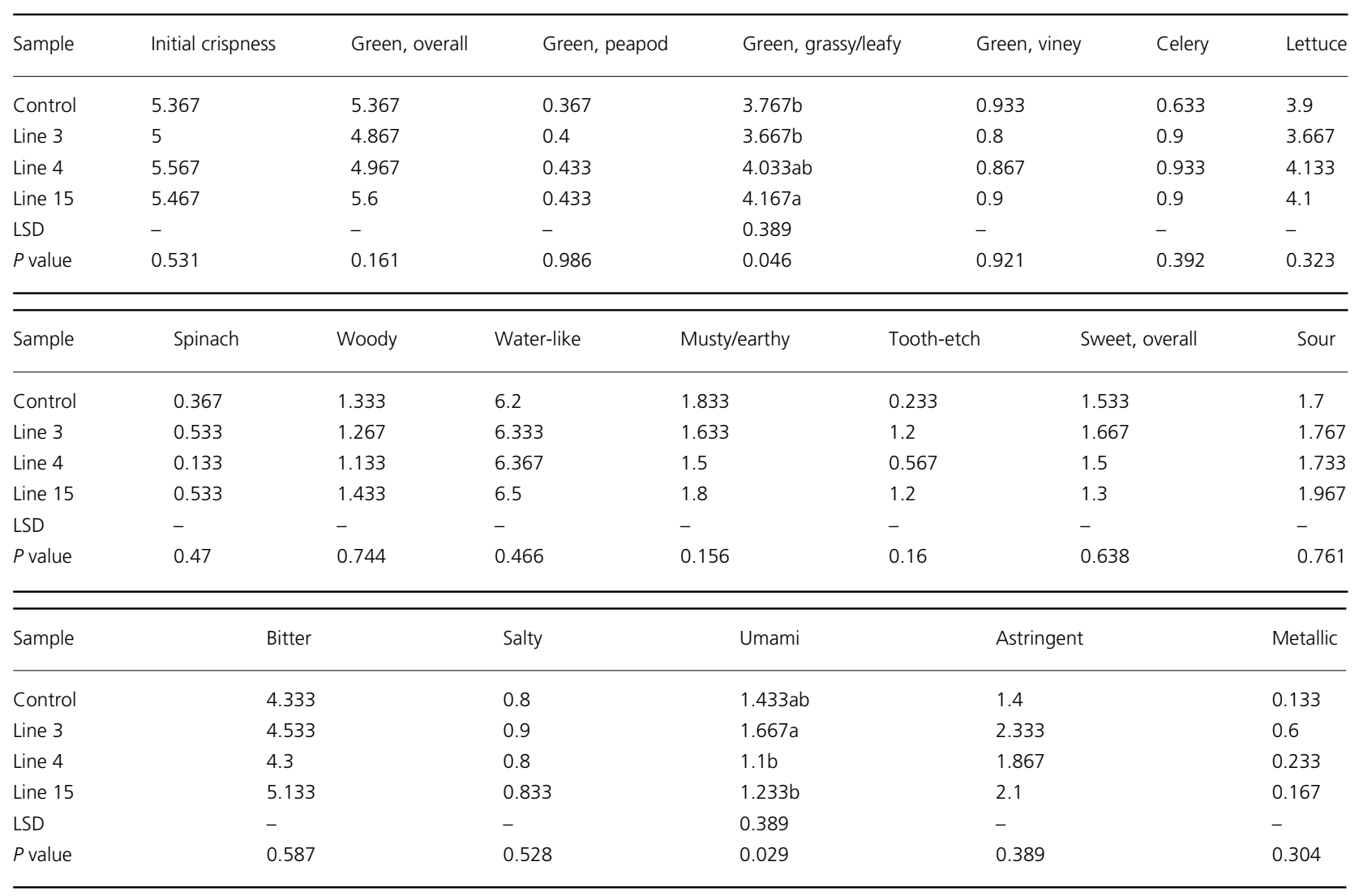

*Mean intensities based on a 15-point scale: 0 , none; 15, extremely strong. Samples with different letters within the attribute column are significantly different at the $95 \%$ confidence level. 
controls with only a $<0.5$ difference (on a scale of $0-15$ ) in intensity. Indeed, although the trained panel were able to find these small differences, it is doubtful whether this would be of practical importance to consumers.

\section{Discussion}

Most Americans do not obtain enough calcium in their diet. To help compensate for this deficiency, one strategy is to increase the calcium content of the foods that they do eat. In this study, we have shown that it is possible to increase the calcium content of lettuce; when applied to a wide variety of fruits and vegetables, this could lead to greater calcium consumption in the diet. We have demonstrated that the total calcium per serving of lettuce is $25 \%-32 \%$ higher in sCAX1 lettuce than in controls (14.5 vs. $18.1-19.2 \mathrm{mg} \mathrm{Ca} / \mathrm{g}$ dry weight, $P<0.001)$.

The sensory analysis of biofortified foods has not been studied extensively; however, this is an important component in the development of any new food. Texture and taste help to form our relationships with food. Everyone has a different sensitivity to certain tastes and flavours, but also to the texture and mouth-feel of foods. Some calcium forms have a better taste and mouth-feel than others, such that some calcium salts are considered as tasteless, whereas others tend to be gritty. For example, calcium citrate is quite acidic and has a bitter twang, whereas calcium carbonate has a soapy taste (Lawless et al., 2003). Thus, it was interesting to determine whether sCAX1-expressing calcium-biofortified lettuce would have different taste and flavour components from controls. Professional descriptive panellists determined the attributes relevant to flavour, bitterness and crispness of control and sCAX1-expressing biofortified lettuce. The flavour of calciumfortified lettuce was virtually identical to that of controls, and no effect was noted on bitterness and crispness. Consumer acceptance of sCAX1-expressing lettuce should not be affected by a change in flavour, bitterness or crispness because only minimal differences were observed. We anticipate that fortified lettuce contains increased total levels of dietary calcium that will translate into improved bioavailability and metabolic usefulness. Feeding trials using sCAX1expressing carrots demonstrated that the total amount of calcium absorbed was increased significantly in both mice and humans with diets containing the modified carrots (Morris et al., 2008). Interestingly, not all of the increased calcium in the transporter-modified carrots was bioavailable. It will be interesting to observe whether this trend is maintained in other sCAX1-expressing plants, such as lettuce.
Although Americans eat lettuce regularly, the increased amounts of calcium present in the SCAX1-expressing lines characterized here will only alleviate marginally the calcium deficiencies of consumers. However, this technology in parallel with additional calcium supplementation during the lettuce growth cycle may substantially boost calcium levels in these transgenic plants. In previous studies, we have demonstrated that growing plants in the presence of high levels of $\mathrm{CaCl}_{2}$ can further increase the amount of calcium in the plant matrices of sCAX1-expressing lines (Hirschi, 1999; Shigaki and Hirschi, 2006). Moreover, it is possible that higher sCAX1 expression may induce a further increase in calcium accumulation in lettuce if exogenous calcium (e.g. $\mathrm{CaCl}_{2}$ ) is added to soil (Park et al., 2004). Future work will address the ability to significantly improve the calcium content of lettuce.

In addition to the nutritional benefits, the use of genetic engineering to increase calcium levels could improve lettuce productivity and extend product shelf life. Calcium is associated with the maintenance of the cell wall structure of vegetables by interacting with pectin to form calcium pectate, and is reported to maintain firmness by generating cross-links with non-esterified pectins in the primary cell wall and middle lamella (Jarvis, 1984; Poovaiah et al., 1988). Thus, fruit and vegetables treated with calcium are generally firmer than controls during storage as a result of increased calcium levels in the hypodermal mesocarp tissue (Camire et al., 1994; Lester and Grusak, 1999). Calcium has long been used as a firming agent for fruits and vegetables, such as cantaloupes, strawberry and carrots, to combat many post-harvest issues (Morris et al., 1985; Luna-Guzman et al., 1999; MartinDiana et al., 2005). Apples are also immersed in a calcium solution to maintain firmness in shipping and to prolong shelf life (Raybaudi-Massiliaa et al., 2007). Recently, sCAX1 expression has been shown to increase calcium levels in tomatoes and increase fruit firmness and prolong shelf life (Park et al., 2005b). Given these examples, the use of sCAX1 expression in lettuce could impact on both plant productivity and human nutrition. Tests of the shelf life qualities of sCAX1-expressing lettuce are in progress.

Our findings evaluated directly the taste qualities of transgenic foods. We established unequivocally that the modification of a single plant calcium transporter increased the calcium content without having a negative impact on lettuce quality. Although this work represents studies towards the commercialization of sCAX1-expressing lettuce, our scientific approach should be applicable to numerous other biofortified crops. 


\section{Experimental procedures}

\section{Plant material, transformation and growth conditions}

Lettuce (Lactuca sativa L. var. Simpson) transformation was performed via Agrobacterium-mediated transformation using leaf disc explants. Seeds were surface-sterilized and germinated on MS inorganic salt medium (Murashige and Skoog, 1962) with $30 \mathrm{~g} / \mathrm{L}$ sucrose, pH 5.7, and solidified using $8 \mathrm{~g} / \mathrm{L}$ agar (PhytoTechnology, Shawnee Mission, $\mathrm{KS}, \mathrm{USA}$ ). The lettuce leaves after 6 weeks of growth in vitro were excised and cultured on MS inorganic salts with $100 \mathrm{mg} / \mathrm{L}$ inositol, MS vitamins, $30 \mathrm{~g} / \mathrm{L}$ sucrose, $2 \mathrm{mg} / \mathrm{L} \mathrm{N}$-6(2-isopentenyl)-adenine, $0.1 \mathrm{mg} / \mathrm{L}$ indole acetic acid and $8 \mathrm{~g} / \mathrm{L}$ agar. At the end of the 1-day preculture, the leaves were dipped in an Agrobacterium culture, blotted and re-cultured on the same medium for $72 \mathrm{~h}$. Leaf sections were then cultured on a selection medium containing MS inorganic salts, $30 \mathrm{~g} / \mathrm{L}$ sucrose, $100 \mathrm{mg} / \mathrm{L}$ inositol, MS vitamins, $0.4 \mathrm{mg} / \mathrm{L} \mathrm{6-}$ benzyl-aminopurine, $0.05 \mathrm{mg} / \mathrm{L}$ naphthaleneacetic acid, $100 \mathrm{mg} / \mathrm{L}$ kanamycin, $250 \mathrm{mg} / \mathrm{L}$ Clavamox ${ }^{\circledR}$ and $8 \mathrm{~g} / \mathrm{L}$ agar. Cultures were maintained at $22{ }^{\circ} \mathrm{C}$ under a 14 -h photoperiod. After $6-8$ weeks (subcultured once at 3-4 weeks), regenerated shoots were transferred to rooting medium for six more weeks, and then established in soil. All plants were watered as needed. Once a week they were watered with Miracle-GroR for tomato (Scotts Miracle-Gro Products, Port Washington, NY, USA). The temperature of the growth chamber was maintained within the range $16-18{ }^{\circ} \mathrm{C}$ under a $14-\mathrm{h}$ photoperiod.

\section{Bacterial strain and plasmid}

The sCAX1 open reading frame was cloned into the nos/nptll/ nos-ter/cdc2a/nos-ter expression vector, which was obtained from John Celenza (Boston University, Boston, MA, USA; Doerner et al., 1996). The plasmids, pcdc2A::sCAX1 and pCaMV35S::sCAX1 (Hirschi, 1999), were introduced into Agrobacterium tumefaciens strain LBA4404 (Hoekema et al., 1983) using the freeze-thaw method (Holsters et al., 1978).

\section{DNA isolation, PCR and Southern blot analysis}

Lettuce genomic DNA was extracted from leaf tissue using a DNeasy Plant Kit (Qiagen, Hilden, Germany), according to the manufacturer's instructions. The 5' (ATGTCTTCTTCTTCTTTGAG) and $3^{\prime}$ (CAATGTAGCTGATCAACATAAC) primers in the $S C A X 1$ coding region were used as primers to amplify a 640-bp fragment, which demonstrates the presence of transformed foreign DNA. PCRs were performed in $25 \mu \mathrm{L}$ of reaction mixture containing $0.2 \mu \mathrm{g}$ of template, $2.5 \mu \mathrm{L}$ of $10 \times$ PCR buffer, $50 \mathrm{pmol}$ of each primer, $5 \mu \mathrm{L}$ of deoxynucleoside triphosphate (dNTP) ( $0.1 \mathrm{~mm}$ ) and 1.25 units of Taq DNA polymerase (Roche Applied Science, Indianapolis, IN, USA). PCR amplification was conducted with an initial denaturation at $94^{\circ} \mathrm{C}$ for $2 \mathrm{~min}$, followed by 30 cycles of $94^{\circ} \mathrm{C}$ for $30 \mathrm{~s}, 50^{\circ} \mathrm{C}$ for $1 \mathrm{~min}$ and $72{ }^{\circ} \mathrm{C}$ for $1 \mathrm{~min}$ $30 \mathrm{~s}$, and then a final 7 -min extension at $72^{\circ} \mathrm{C}$. The resulting PCR products were examined by running on a $1.2 \%(\mathrm{w} / \mathrm{v})$ agarose gel using an electrophoresis system. Southern blot analysis was carried out as described previously (Park et al., 2005b). DNA (5-10 $\mu \mathrm{g}$ ) was digested with Xbal (for pCaMV35S::sCAX1) or EcoRI (for pcdc2A::sCAX1), separated in a $0.9 \%(\mathrm{~W} / \mathrm{V})$ agarose gel by electrophoresis and blotted on to a nylon membrane (Zeta-Probe GT membrane, BIORAD
Laboratories, Hercules, CA, USA). The probe for the sCAX1 gene was isolated from a Notl (1.4-kb) restriction fragment of the p039 plasmid (Hirschi, 1999). The membranes were pre-hybridized overnight at $65^{\circ} \mathrm{C}$ in $7 \%$ sodium dodecylsulphate (SDS) and $0.25 \mathrm{M} \mathrm{Na}_{2} \mathrm{HPO}_{4}$, and then hybridized overnight at $65^{\circ} \mathrm{C}$ in the same solution containing the probe labelled with ${ }^{32} \mathrm{P}-\mathrm{dCTP}$ using a NEBlot Kit (NEB BioLabs, Beverly, MA, USA). Membranes were washed twice for 30 min each with $20 \mathrm{mM} \mathrm{Na}_{2} \mathrm{HPO}_{4}$ and $5 \% \mathrm{SDS}$ at $65^{\circ} \mathrm{C}$, and then washed twice again for 30 min each with $20 \mathrm{mM} \mathrm{Na}_{2} \mathrm{HPO}_{4}$ and $1 \% \mathrm{SDS}$ at $65^{\circ} \mathrm{C}$. Membranes were exposed to $\mathrm{X}$-ray film at $-80^{\circ} \mathrm{C}$.

\section{RNA isolation, RT-PCR and Northern blot analysis}

Total RNA was extracted from leaves using an RNeasy Plant Kit (Qiagen), according to the manufacturer's instructions. Total RNA $(7 \mu \mathrm{g})$ was separated on a $1.2 \%$ agarose gel containing $1.5 \%$ formaldehyde, and blotted on to a Zeta-Probe GT membrane according to the manufacturer's instructions. Hybridization and washing were as described previously in Southern blot analyses. RNA for RT-PCR was treated with an RNase-free DNase set (Qiagen) prior to the synthesis of first-strand CDNA by oligo(dT) priming using Moloney murine leukaemia virus-reverse transcriptase (BD Biosciences Clontech, Palo Alto, CA, USA). PCR amplification for RT-PCR was performed in $25 \mu \mathrm{L}$ of reaction mixture with an initial denaturation at $94{ }^{\circ} \mathrm{C}$ for $2 \mathrm{~min}$, followed by 30 cycles of $94{ }^{\circ} \mathrm{C}$ for $30 \mathrm{~s}, 50^{\circ} \mathrm{C}$ for $1 \mathrm{~min}$ and $72^{\circ} \mathrm{C}$ for $1 \mathrm{~min} 30 \mathrm{~s}$, and then a final 7 -min extension at $72^{\circ} \mathrm{C}$.

\section{Calcium and mineral analysis}

Harvested lettuce leaves at 60 days after germination were dried at $70{ }^{\circ} \mathrm{C}$ for 4 days, and a total of $0.25 \mathrm{~g}$ (dry weight) from each of the lettuce leaves was digested as reported previously (Feagley et al., 1994). Total calcium and mineral contents per gram of dry weight were determined using an inductively coupled plasma emission spectrophotometer (Spectro, Kleve, Germany). For the $T_{1}$ transgenic plants, the lettuce leaves (at least $\sim 50 \mathrm{~g}$ ) from each of 16 transgenic lines (eight 35SCAX lines and eight CDCCAX lines) and four controls were harvested, dried, and calcium and mineral analyses were conducted on individual lines. Segregation analysis on $T_{2}$ seeds from self-pollinated $T_{1}$ transgenic plants (35SCAX-3, 35SCAX-4 and CDCCAX-15) were carried out on $100 \mathrm{mg} / \mathrm{L}$ kanamycin selection medium, and homozygous $T_{2}$ lines were selected for subsequent analysis. For the $T_{2}$ transgenic plants, the lettuce leaves (at least $\sim 50 \mathrm{~g}$ ) from each homozygous $\mathrm{T}_{2}$ line derived from three $T_{1}$ transgenic plants (35SCAX-3, 35SCAX-4 and CDCCAX-15) were harvested and analysed as described above.

\section{Yield measurements}

At harvest, all growth chamber-grown lettuce leaves were harvested and weighed individually. For yield measurements, the fresh weight of the leaves of $s C A X 1$-expressing lettuces was obtained from the means of six different progeny plants from 35SCAX-3, 35SCAX-4, CDCCAX-15 and control lines.

\section{Statistical analysis}

All data were analysed as a completely randomized design using the SAS procedure GLM (version 9.00; SAS Institute Inc., Cary, NC, USA). 
Table 3 Lettuce attributes, definitions, references and intensities on a 15-point scale

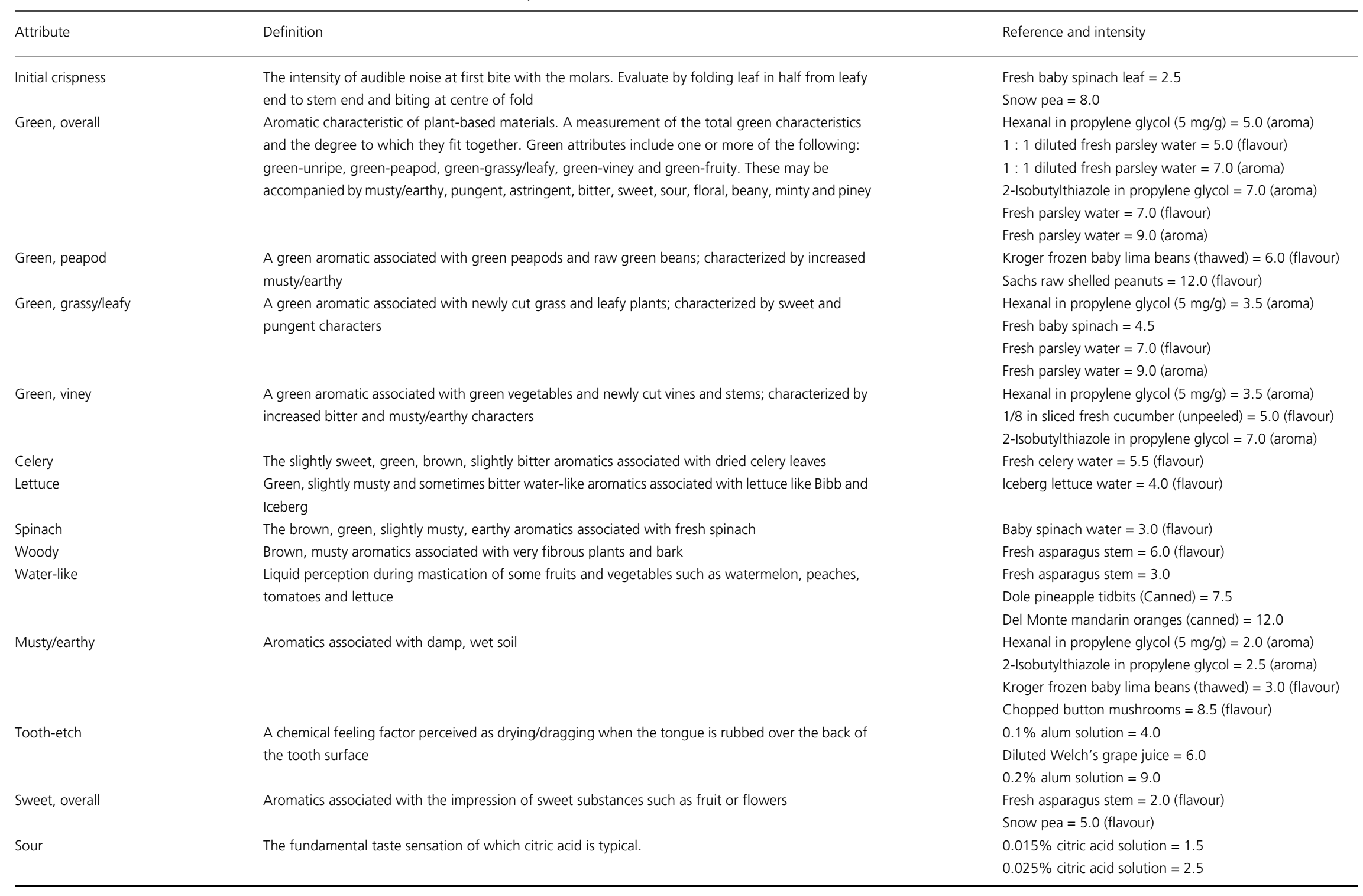




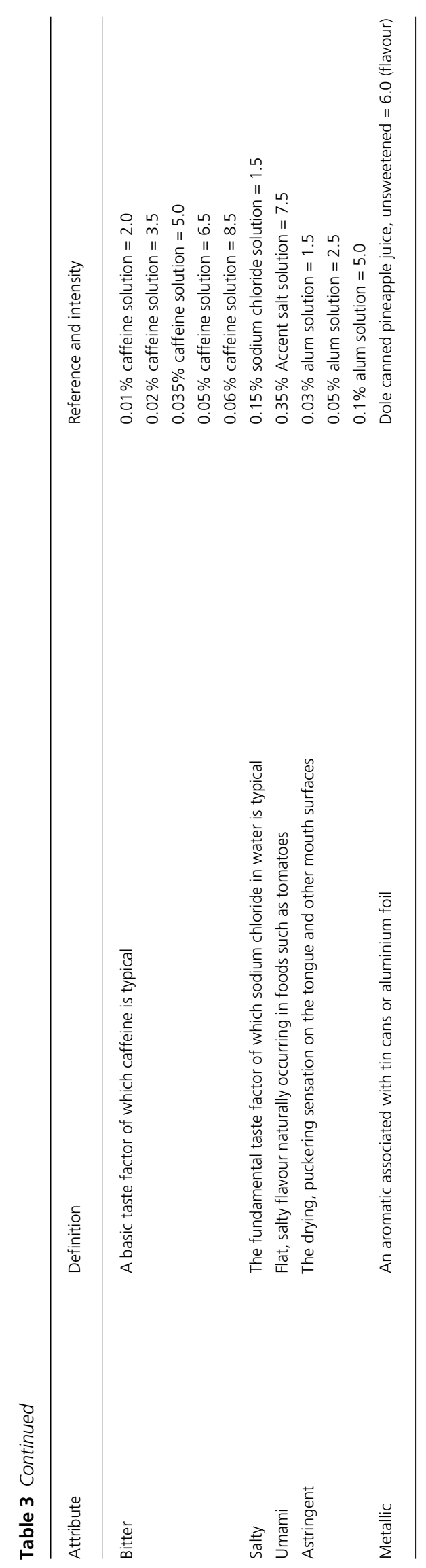

Dunnett's test (Dunnett, 1955) was computed (at the 0.05 probability level) to compare treatment with reference. The data reported represent the mean of at least three replications.

\section{Sensory analysis}

Five highly trained panellists from the Sensory Analysis Center (Kansas State University, Manhattan, KS, USA) were selected to perform the sensory analysis of calcium-biofortified lettuce. All panellists had completed $120 \mathrm{~h}$ of sensory descriptive training, had more than $1500 \mathrm{~h}$ of sensory panel testing experience on a wide range of products, and had previous experience of testing vegetables, including leafy green vegetables. At 60 days after germination, growth chamber-grown lettuces from three different progeny plants $\left(T_{2}\right)$ of 35SCAX-3, 35SCAX-4, CDCCAX-15 and control lines were harvested and rinsed free of soil.

Lettuces were stored in plastic bags in the refrigerator at $4{ }^{\circ} \mathrm{C}$ until use. Immediately prior to serving, the appropriate sample was removed from the refrigerator, and leaves were cut/torn off. Two leaves from a plant were served to each panellist on a $15-\mathrm{cm}$ round foam plate coded with a three-digit code.

The method for lexicon development was adapted from the profile methods of flavour analysis (Keane, 1992), and was similar to that used in recent sensory studies (Karagul-Yuceer et al., 2007; Lee and Chambers, 2007; Yates and Drake, 2007). Attributes and reference standards from previous studies of leafy green vegetables and associated studies (e.g. green flavour; Hongsoongnern and Chambers, 2008) were provided to the panellists. Panellists were also provided with lettuces representing a range of potential samples. The panellists discussed the possible flavour of the lettuce samples and determined the flavour attributes relevant to this specific lettuce study. Initial crispness was also added to minimally address the texture of the samples. The definitions and reference standards for the additional attributes were agreed upon, and the ballot order was established. The panel spent a total of $2 \mathrm{~h}$ reviewing potential attributes and establishing the lexicon for this study. Attributes, definitions and references are shown in Table 3. The panel evaluated samples one at a time following a randomized complete block design with replication as the blocking factor. Four samples (one control and three calcium-biofortified lines) were tested in a 1-h period.

Panellists evaluated individually the intensities of each attribute using the lexicon and techniques developed during orientation. Data were collected using a computerized data collection system (Compusense Five version 4.4.8, 2002, Guelph, ON, Canada). Intensities were scored on a 15 -point intensity scale divided into 0.5 -point increments ( 0 , none; 15 , extremely strong). Reverse osmosis, deionized, carbon-filtered water and unsalted crackers were used to cleanse the palate between samples.

\section{Statistical analysis of sensory evaluation}

Data were analysed in two ways: first, by comparing all test lines as a group with the control line to determine whether a difference existed between control and calcium-biofortified lines; second, by comparing all lines individually to determine whether there were any differences between the various calcium-biofortified lines compared with the control line. Analysis was conducted in SAS (version 9.13) 
using analysis of variance (Proc Glimmix and Fisher's protected leastsignificant difference) at the $95 \%$ confidence level to determine significant differences between specific lettuces.

\section{Acknowledgements}

This research was supported by the following: the Kansas State University AES project (NAHF381121) and NHRI RDA-KSU Cooperative Research Project to SHP; the US Department of Agriculture/Agricultural Research Service (under Cooperative Agreement 58-62650-6001), National Institutes of Health Grant 1R01 DK 062366 and USDACSREES grant 2005-34402-6401 Designing Foods for Health to $\mathrm{KDH}$; and the National Institutes of Health STTR Phase I grant 1 R41 AR052225-01 A1 to MPE.

\section{References}

Bouis, H.E. (2003) Micronutrient fortification of plants through plant breeding: can it improve nutrition in man at low cost? Proc. Nutr. Soc. 62, 403-411.

Camire, M.E., Ismail, S., Work, T.M., Bushway, A.A. and Halteman, W.A. (1994) Improvements in canned lowbush blueberry quality. J. Food Sci. 59, 394-398.

Diaz de la Garza, R.I., Gregory III, J.F. and Hanson, A.D. (2007) Folate biofortification of tomato fruit. Proc. Natl. Acad. Sci. USA, 104, 4218-4222.

Doerner, P., Jorgensen, J.-E., You, R., Steppuhn, J. and Lamb, C. (1996) Control of root growth and development by cyclin expression. Nature, 380, 520-523.

Dunnett, C.W. (1955) A multiple comparison procedure for comparing several treatments with a control. J. Am. Statist. Assoc. 50, 1096-1121.

Feagley, S.E., Valdez, M.S. and Hudnall, W.H. (1994) Papermill sludge, phosphorus, potassium, and lime effect on clover grown on a mine soil. J. Environ. Qual. 23, 759-765.

Feskanich, D., Weber, P., Willett, W.C., Rockett, H., Booth, S.L. and Colditz, G.A. (1999) Vitamin K intake and hip fractures in women: a prospective study. Am. J. Clin. Nutr. 69, 74-79.

Guegen, L. and Pointillart, A. (2000) The bioavailability of dietary calcium. J. Am. Coll. Nutr. 19, 119S-136S.

Hirschi, K.D. (1999) Expression of Arabidopsis CAX1 in tobacco: altered calcium homeostasis and increased stress sensitivity. Plant Cell, 11, 2113-2122.

Hoekema, A., Hirsch, P.R., Hooykaas, P.J.J. and Schillperoort, R.A. (1983) A binary plant vector strategy based on separation of vir- and T-region of the Agrobacterium tumefaciens Ti-plasmid. Nature, 303, 179-180.

Holsters, M., De Waele, D., Depicker, A., Messens, E., Van Montagu, M. and Schell, J. (1978) Transfection and transformation of A. tumefaciens. Mol. Gen. Genet. 163, 181-187.

Hongsoongnern, P. and Chambers, E.I.V. (2008) A lexicon for green odor or flavor and characteristics of chemicals associated with green. J. Sens. Stud. 23, 205-221.

Jarvis, M.C. (1984) Structure and properties of pectin gels in plant cell walls. Plant Cell Environ. 7, 153-164.
Karagul-Yuceer, Y., Isleten, M. and Uysal-Pala, C. (2007) Sensory characteristics of Ezine cheese. J. Sens. Stud. 22, 49-65.

Keane, P. (1992) The flavor profile. In: Manual on Descriptive Analysis Testing for Sensory Evaluation, Vol. 13 (Hootman, R.C., ed.), pp. 5-21. West Conshohocken, PA: Manual Series MNL.

Lawless, H.T., Rapacki, F., Horne, J. and Hayes, A. (2003) The taste of calcium and magnesium salts and anionic modifications. Food Qual. Prefer. 4, 319-325.

Lee, J. and Chambers, D.H. (2007) A lexicon for flavor descriptive analysis of green tea. J. Sens. Stud. 22, 256-272.

Lester, G.E. and Grusak, M.A. (1999) Postharvest application of calcium and magnesium to honeydew and netted muskmelons: effects on tissue ion concentrations, quality, and senescence. J. Am. Soc. Hortic. Sci. 124, 545-552.

Luna-Guzman, I., Cantwell, M. and Barrett, D.M. (1999) Fresh-cut cantaloupe: effects of $\mathrm{CaCl}_{2}$ dips and heat treatments on firmness and metabolic activity. Postharvest Biol. Technol. 17, 201-213.

Martin-Diana, A.B., Rico, D., Barry-Ryan, C., Frias, J., Mulcahy, J. and Henehan, G.T.M. (2005) Comparison of calcium lactate with chlorine as a washing treatment for fresh-cut lettuce and carrots: quality and nutritional parameters. J. Sci. Food Agric. 51, 391394.

Morris, J.R., Sistrunk, W.A., Sims, C.A., Main, G.L. and Wehunt, E.J. (1985) Effects of cultivar, postharvest storage, pre-processing dip treatments and style of pack on the processing quality of strawberries. J. Am. Soc. Hortic. Sci. 110, 172-177.

Morris, L., Hawthorne, K.M., Hotze, T., Abrams, S.A. and Hirschi, K.D. (2008) Nutritional impact of elevated calcium transport activity in carrots. Proc. Natl. Acad. Sci. USA, 105, 1431-1435.

Murashige, T. and Skoog, F. (1962) A revised medium for rapid growth and bioassays with tobacco tissue culture. Physiol. Plant. 15, 473-497

National Academy of Sciences (1999) Dietary Reference Intakes for Calcium, Phosphorus, Magnesium, Vitamin D and Fluoride. Washington DC: Institute of Medicine, National Academy Press.

Park, S.H., Kim, C.-K., Pike, L.M., Smith, R.H. and Hirschi, K.D. (2004) Increased calcium in carrots by expression of an Arabidopsis $\mathrm{H}^{+}$/ $\mathrm{Ca}^{2+}$ transporter. Mol. Breed. 14, 275-282.

Park, S.H., Kang, T.-S., Kim, C.-K., Han, J.-S., Kim, S., Smith, R.H., Pike, L.M. and Hirschi, K.D. (2005a) Genetic manipulation for enhancing calcium content in potato tuber. J. Agric. Food Chem. 53, 5598-5603.

Park, S.H., Cheng, N.H., Pittman, J.K., Yoo, K.S., Park, J., Smith, R.H. and Hirschi, K.D. (2005b) Increased calcium levels and prolonged shelf life in tomatoes expressing Arabidopsis $\mathrm{H}^{+} / \mathrm{Ca}^{2+}$ transporters. Plant Physiol. 139, 1194-1206.

Pfeiffer, W.H. and McClafferty, B. (2007) Biofortification: breeding micronutrient-dense crops. In: Breeding Major Food Staples (Kang, M.S., ed.). pp 61-91. Oxford: Blackwell Science Ltd.

Poovaiah, B.W., Glenn, G.M. and Reddy, A.S.N. (1988) Calcium and fruit softening: physiology and biochemistry. Hortic. Rev. 10, 107-152.

Raybaudi-Massiliaa, R.M., Mosqueda-Melgara, J., Sobrino-Lópeza, A., Soliva-Fortunya, R. and Martín-Belloso, O. (2007) Shelf-life extension of fresh-cut 'Fuji' apples at different ripeness stages using natural substances. Postharvest Biol. Technol. 45, 265-275.

Shigaki, T. and Hirschi, K.D. (2006) Diverse functions and molecular properties emerging for $\mathrm{CAX}$ cation $/ \mathrm{H}^{+}$exchangers in plants. Plant Biol. 8, 419-429. 
United States Department of Agriculture (2000) Continuing Survey of Food Intakes by Individuals (CSFII), 1994-1996. URL http:// www.barc.usda.gov/bhnrc/foodsurvey/home.htm [accessed 25 July 2006]

United States Department of Agriculture (2004) USDA National
Nutrient Database for Standard Reference, Release 16-1. URL Nutrient Data Laboratory Home Page http://www.nal.usda.gov/ fnic/foodcomp [accessed 1 March 2006].

Yates, M.S. and Drake, M.A. (2007) Texture properties of Gouda cheese. J. Sens. Stud. 22, 493-506. 